\title{
Wissen, was passiert ist
}

Datenschutz dient im Kern dem Ziel, freien Bürgern zu ermöglichen, unbefangen und in Herrschaft über die eigenen Daten zu handeln und zu kommunizieren. Die Datensparsamkeit ist eines der vornehmsten Datenschutzprinzipien, und am sparsamsten ist die Unverfolgbarkeit von Kommunikation. Auf der anderen Seite will und sollte man aber genau nachvollziehen können, was passiert ist, wenn etwas schief geht. Mit dem Hinweis auf Kinderpornographie und terroristische Bedrohung wirbt der Staat bei den Bürgern darum, zu ihrer Sicherheit den Strafverfolgungsbehörden zuzugestehen, Mechanismen zur Aufdeckung von Kommunikationsspuren einzusetzen. Und nicht nur der Staat bedarf der Spurenverfolgung: auch Firmen und Organisationen erheben Anspruch darauf, Missbrauch ihrer Kommunikationsinfrastrukturen zu unterbinden. Auf der einen Seite also Datenvermeidung, auf der anderen Seite Spurensicherung - wie soll das zusammengehen? Wie bei allen Wertkonflikten kann das nur durch klare Grenzziehungen und saubere Verfahren gelingen.

Dieses Heft befasst sich mit den Mechanismen, Möglichkeiten und Grenzen von Spurenverfolgung im Netz. Im gerichtlichen Kontext nennt man das „Forensik“, aus dem Lateinischen „Forum“ für Marktplatz und Öffentlichkeit: vor Gericht ist in aller Öffentlichkeit unzweifelhaft aufzudecken, was Böses passiert ist. Für die Informationstechnik und ihre Anwendungen unterscheiden wir innerhalb der IT-Forensik die Computerforensik', bei der die Geräte daraufhin untersucht werden, was auf ihnen gespeichert ist und was auf ihnen ggf. sogar vor Löschvorgängen gespeichert war, von der Kommunikationsforensik, bei der Verkehrsdaten analysiert werden, einerseits rückblickend aus Logging-Daten, andererseits aktuell aus Live-Mitschnitten.

Selbstverständlich sind der Spurensicherung Grenzen gesetzt. Dennis Heinson, York Yannikos, Frederik Franke, Christian Winter und Markus Schneider von der Universität Kassel bzw. dem Fraunhofer-Institut für Sichere Informationstechnik SIT in Darmstadt behandeln im ersten Beitrag dieses Heftes rechtliche Fragen zur technischen Praxis IT-forensischer Analysen; damit begeben sie sich genau in das oben angesprochene Spannungsfeld zwischen Datenschutz und Missbrauchsanalyse.

Matthias Bäcker, Felix C. Freiling und Sven Schmitt von der Universität Mannheim hinterfragen in ihrem Beitrag die Gründe dafür, dass heutzutage in der Regel aufwändige 1:1-Kopien des originalen Beweismittels erstellt werden. Sie schlagen Alternativen vor, mit denen man Speichermedien effizienter und trotzdem gerichtsfest sichern kann.

Rüdiger Grimm und Daniel Pähler von der Universität in Koblenz widmen sich in ihrem Beitrag dem Spezialgebiet der E-Mail-Forensik, in dem zur Aufklärung von Straftaten ermittelt wird, wer mit wem von welchem Ort aus und wann über welche Inhalte E-Mails ausgetauscht hat; ein Gebiet, das in der modernen Praxis des Strafrechts zunehmend an Bedeutung gewinnt, zuletzt in den großen Terroristenprozessen vor dem Oberlandesgericht in Koblenz.

Eine Forschergruppe des Fraunhofer-Instituts für Sichere Informationstechnik SIT in Darmstadt um York Yannikos, Christian Winter, Frederik Franke und Markus Schneider stellt ein Verfahren zur Erzeugung von synthetischen Daten vor, die für den Test IT-forensischer Werkzeuge geeignet sind. Damit vermeiden sie erstens den zweckfremden Umgang mit personenbezogenen Daten, und zweitens können sie Testdaten gezielt so strukturieren, dass sie Schwächen von Testwerkzeugen aufdecken.

Schließlich zeigen Philipp Zikesch und Bernd Reimer von der PricewaterhouseCoopers AG in Stuttgart in ihrem Positionspapier auf, dass die Novellierung des BDSG keinen Zielkonflikt zwischen Datenschutz und präventiver Korruptionsbekämpfung erzeugt. Das bedeutet nach ihrer Überzeugung, dass präventive Untersuchungsmaßnahmen zur Aufdeckung von Straftaten im Unternehmen unter den gleichen Zulässigkeitsvoraussetzungen möglich sind wie vor der BDSG-Novellierung.

\section{Rüdiger Grimm}

1 Siehe auch das Gateway in DuD 8/2004, S. 491. 